\title{
PW10-02
}

\section{MEDED - A NOVEL INTERACTIVE MANUAL TO ENHANCE PSYCHOPHARMACOLOGIC CARE IN CHILDREN AND ADOLESCENTS}

\section{S. Kutcher}

Psychiatry, Dalhousie University, Halifax, Canada

Child and Adolescent psychopharmacology is a complex yet frequently necessary psychiatric intervention that requires the participation of the patient, the family, other caregivers, other health providers as well as physicians. Provision of up to date information about and education in the use of psychotropics is an essential component of good psychopharmacologic treatment. To date there have been no methods of addressing these needs within a collaborative care framework that meets the interests of all parties and optimizes the application of best available knowledge in the clinical care of child and adoelscents with mental disorders. MEDED (C) was developed by a group of pharmacists and child psychiatrists as an innovative tool to meet this need. It has been extensively evaluated in a variety of clinical settings in Canada with substantial positive results. This presentation will review the novel collaborative approach to pharmacotherapy that MEDED supports, the process of MEDED development, the contents of MEDED and research on its use to date. Clinicians and program directors will have the opportunity to learn how to obtain further information abot the use of this tool. 